\title{
Gradhiva
}

GRADHIV

Revue d'anthropologie et d'histoire des arts

11 | 2010

Grands hommes vus d'en bas

\section{Grande âme cherche corps sur mesure}

Des animas en général et du sosie de Gandhi en particulier

Great soul seeks made-to-measure body: of animae in general and, more

particularly, of Gandhi's look-alike

\section{Emmanuel Grimaud}

\section{OpenEdition}

\section{Journals}

Édition électronique

URL : http://journals.openedition.org/gradhiva/1656

DOI : 10.4000/gradhiva. 1656

ISSN : $1760-849 x$

\section{Éditeur}

Musée du quai Branly Jacques Chirac

\section{Édition imprimée}

Date de publication : 19 mai 2010

Pagination : $56-79$

ISBN : 978-2-35744-025-8

ISSN : 0764-8928

\section{Référence électronique}

Emmanuel Grimaud, "Grande âme cherche corps sur mesure », Gradhiva [En ligne], 11 | 2010, mis en ligne le 19 mai 2013, consulté le 30 avril 2019. URL : http://journals.openedition.org/gradhiva/1656 ; DOI : 10.4000/gradhiva.1656 


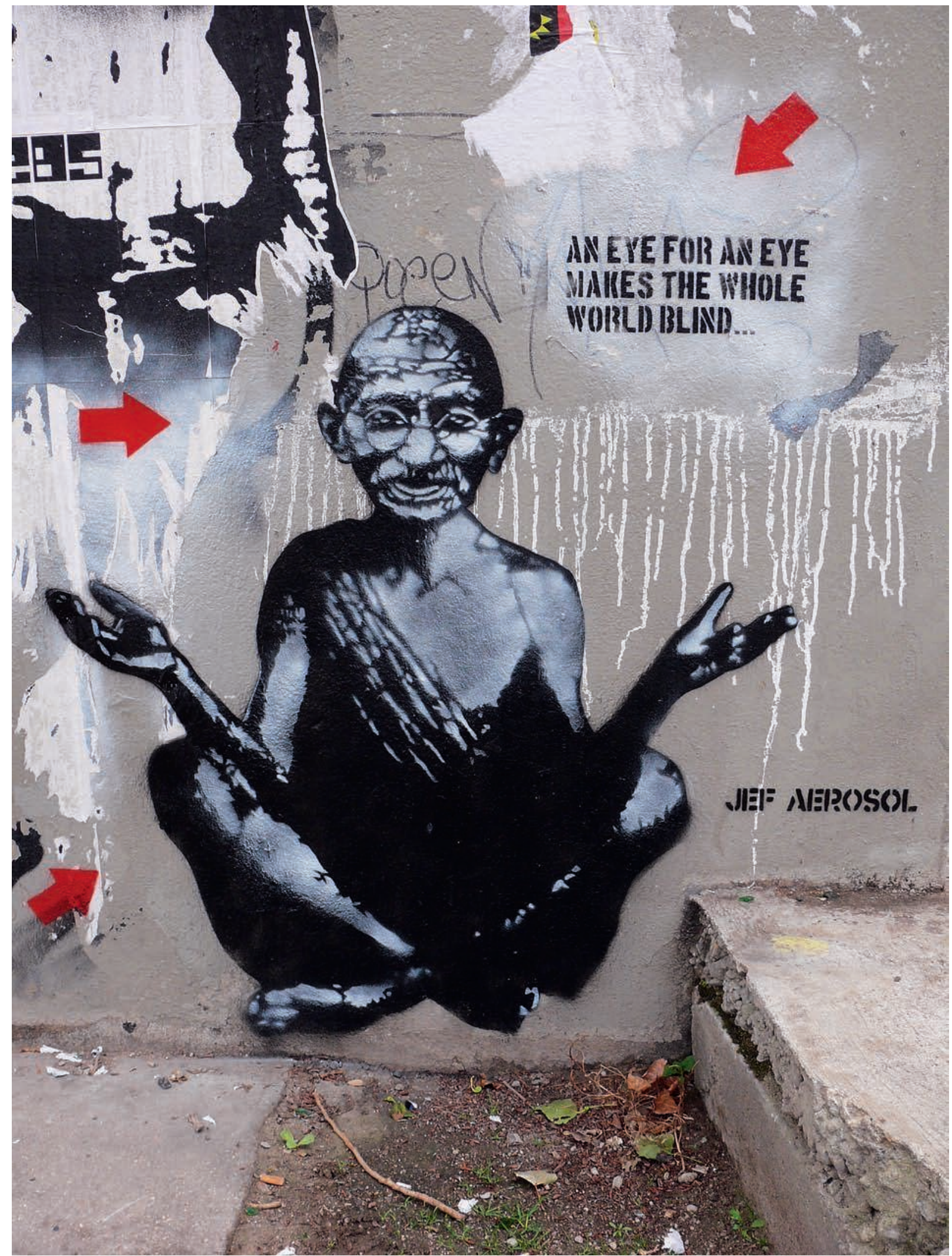

Fig. 1 Pochoir représentant Gandhi, Londres, 2007 @ Jef Aérosol. 
Emmanuel Grimaud

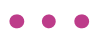

1. On mesure le décalage entre la conception tardienne de la grandeur comme quantité variable et l'approche plutôt rigide du problème qui fut celle d'Émile Durkheim dans Le Rôle des grands hommes dans l'histoire (1883) : «Quoi qu'il en puisse coûter à notre amour-propre, il faut reconnaître que Dieu a fait deux espèces d'hommes bien différentes : il y a les grands, et il y a les petits. » 1975 [1883] : 409]

\section{Grande âme
cherche corps
sur mesure \\ Grande âme
cherche corps
sur mesure \\ Grande âme
cherche corps
sur mesure Des animas en général et
du sosie de Gandhi en particulier Des animas en général et
du sosie de Gandhi en particulier}

Le jour où mes sosies parviendront à contrefaire ma pensée, demanda un jour l'Empereur à un devin, pourrai-je enfin connaître la paix? LE DEVIN - Majesté, je ne le crois pas. Ce jour-là, vous deviendrez un sosie de vos sosies.

Dai Sijie, (2009)

La grandeur d'un homme se mesurerait-elle au nombre de ses sosies? Considérant, avec raison, la gloire comme une quantité variable, le philosophe Gabriel Tarde proposa, dans Psychologie économique (1902), un "gloriomètre» afin de "mesurer avec une certaine approximation, moyennant des statistiques ingénieuses, pour chaque espèce de célébrité, cette quantité singulière ». «La notoriété, écrit-il, est un des éléments de la gloire; elle peut se mesurer facilement par le nombre d'individus qui ont entendu parler d'un homme ou d'un de ses actes. Mais l'admiration, autre élément non moins essentiel, est d'une mesure plus complexe. Il y aurait à la fois à compter le nombre des admirateurs, à chiffrer l'intensité de leurs admirations, et à tenir compte aussi - ce serait là le hic - de leur valeur sociale très inégale [...]. Mais comment préciser numériquement cette supériorité-là? Si ardu que soit ce problème (que certains anthropologistes simplifient fort, en le réduisant à mesurer l'indice crânien, le plus ou moins de dolicho ou de brachycéphalie), il faut bien qu'il soit susceptible d'une solution [...]...» (Tarde 1902 : 56-57) Si on peut regretter que le gloriomètre n'ait pas donné lieu à beaucoup d'applications en économie, on peut se demander dans quelle mesure 
Fig. 2 Cortège funéraire de Gandhi, 31 janvier 1948. (c) Vithalbhai Jhaveri/ GandhiServe.

\section{- $\bullet$}

2. On remarquera que la mort du chanteur Michael Jackson en 2009 (dont on ne sait toujours pas s'il était vraiment dans son cercueil au moment de son enterrement ) a été une aubaine pour le marché des sosies du « roi de la pop ». Ce marché est estimé à plusieurs millions de dollars pour l'industrie du spectacle. De la même façon, comme le souligne le photographe Kamlesh Jemini à propos du sosie de Gandhi, celui-ci n'existerait pas si nous n'avions pas eu de photographie du Mahatma Gandhi.

3. J'ai choisi de mettre à l'épreuve ici d'autres arguments, d'ouvrir d'autres pistes qu'il ne m'a pas été permis d'ouvrir dans le livre. Pour un compte rendu plus complet et détaillé du matériau ethnographique sur lequel je m'appuie, je me permets d'y renvoyer le lecteur. la grandeur d'un individu est fonction du nombre de ses figurations, et même de ses réplications physiques ou du nombre de ses imitations. Serait-ce alors toujours dans ce cas une grandeur que l'on mesure ou bien autre chose, une sorte d'indice de présence, qui lui est certes concomitant mais qui doit être distingué de la première?

On n'a pas encore inventé l'instrument de mesure permettant de naviguer dans la prolifération des figures du même homme afin d'évaluer l'intensité, forte ou faible, de ses représentations et incarnations, d'une peinture par rapport à une sculpture par exemple, d'un corps animé par rapport à une image ou encore d'un buste en marbre par rapport à une figure de cire ou un mannequin en résine. Tous ces modes de matérialisation ne cristallisent pas la même chose, ils n'ont pas non plus, en situation, le même effet de présence. Et lorsqu'ils se multiplient, fusionnent, s'échangent en partie leurs propriétés, il faut s'attendre à voir surgir des figures inédites, voire troublantes. L'histoire d'un sosie de Gandhi, chargé de le faire exister en chair et en os après sa mort, nous aidera peut-être à mieux cerner ce problème de «matériologie » des représentations. Il faudra pour cela répondre à plusieurs questions : pourquoi la mort constitue-t-elle un moment critique, décisif, mais en aucun cas un point final dans la vie d'un grand homme? En quoi un sosie ou une doublure physique constitue-t-il une valeur ajoutée, en termes d'effet de présence, par rapport à une image? Et pour que des répliques physiques d'un individu puissent se multiplier librement sans être trop contraintes par le culte de l'original, à quelle théorie plastique ou figurative faut-il adhérer?

Après la mort, la semi-vie...

Le jour de sa crémation, Gandhi, le Mahatma (littéralement "grande âme »), est devenu un ensemble de traits atemporels et reproductibles pouvant réapparaître à l'infini dans d'autres corps que le sien. Pour réussir sa rematérialisation, il a dû passer un test bien particulier, une épreuve de "sosification ${ }^{2}$ ». Un sosie de Gandhi fut chargé par son entourage, composé de fidèles gandhiens et d'hommes politiques aussi célèbres qu'un ancien président de la République, de faire le Gandhi en chair et en os durant d'innombrables commémorations célébrant le Mahatma. Il ne fait aucun doute que tous les efforts consentis par les fidèles de Gandhi pour le faire exister après sa mort, malgré son absence, se sont faits à son insu. La survivance possède ses propres codes de recomposition et techniques de mise en présence lorsqu'il s'agit des grands hommes, pour les faire exister de manière fantomatique, spectrale, et venir hanter les vivants. Comme nous le verrons, voir un Gandhi se promener dans la rue cinquante ans après la mort du Mahatma ne pose aucun problème aux habitants de Bhopal. Mais avant d'exposer les éléments les plus saillants de cette course à l'effet de présence, détaillée ailleurs ${ }^{3}$ (Grimaud 2007), et dont le sosie de Gandhi fut un point de passage décisif, il nous faut bien comprendre en quoi la mort (ou l'entrée dans la vie post mortem) constitue effectivement un problème qui, s'il est bien surmonté, peut nous conduire au-delà de bien des dualités (inanimé-animé, mort-vivant, présent-absent, matériel-immatériel).

La mort est sans doute le moment le plus délicat dans la vie d'un individu, et la grandeur d'un homme se mesure aussi à la difficulté que son entourage éprouve à l'oublier définitivement. Il n'est pas besoin d'adhérer à la théorie des renaissances pour réaliser qu'à cet instant problématique l'individu entre dans 
un autre régime de présence, une sorte de "semi-vie ", grâce aux supports matériels multiples visant à le faire exister. Sa présence devient ambiguë, flottante, sa survie un enjeu. Plus les supports se multiplient, plus il existe de versions de lui-même, plus se renforce son ambiguïté en tant qu'être. Son décès est généralement le moment où on mesure sa grandeur et où tout une panoplie de dispositifs s'inventent pour le faire durer, obligeant à étendre la notion de biographie au-delà de ses limites ordinaires. Essayons de mieux qualifier ce régime de vie post mortem dans lequel le sosie de Gandhi va nous faire entrer. Philip K. Dick, le célèbre écrivain de science-fiction, est sans doute l'un des grands théoriciens de la semi-vie. Un de ses romans les plus connus, Ubik, publié en 1969, commence dans un moratorium. Glen Runciter, patron d'une société de surveillance chargée de neutraliser le travail d'agences de télépathes (doués de pouvoirs psioniques et qui font de l'espionnage industriel), vient y rendre visite à sa femme, Ella, maintenue en état de semi-vie dans un cercueil de verre à la connectique impressionnante. Son mari lui parle par une sorte de téléphone, mais la connexion est assez mauvaise et constamment parasitée car la présence de son voisin (lui aussi en semi-vie) produit des interférences. Runciter est venu prendre des conseils de sa femme, qui joue un rôle important dans la moindre de ses décisions : au moment de mourir, Ella lui a demandé de garder le contrôle de son entreprise. Puis survient l'inévitable : un attentat visant Runciter et tous les membres de son entreprise. Ces derniers croient avoir survécu à l'attentat et pensent que leur patron est mort. Ils le conduisent au moratorium où se trouve sa femme et essaient de le mettre en semi-vie : cette technologie permet de prolonger l'agonie et d'étendre les derniers instants de la conscience, mais n'est praticable que si la personne est encore vivante. Le lecteur s'aperçoit plus loin qu'en réalité tous ceux qui ont cru échapper à l'attentat sont morts et survivent en état de semi-vie alors que Runciter, lui, est bien vivant. Les personnages circulent donc dans le monde fantasmatique des semi-vivants, un drôle de monde où tout régresse étrangement. Ignorant leur nouvelle condition, les protagonistes voient ce qu'ils acceptent comme la réalité se déliter progressivement. Le monde se met à remonter dans le temps, des anachronismes se font jour, des personnes meurent sans raison apparente. Le plus étonnant réside sans doute dans les messages de Runciter luimême : "Je suis vivant, vous êtes morts. " Ils interviennent de façon sporadique tout au long du roman, sous forme de graffitis ou de publicités, et sont supposés aider les autres personnages à survivre.

Version high tech de réincarnation où les individus, après avoir été semivivants, peuvent exister ailleurs dans une autre matrice et dans un autre corps, la technologie imaginée par Dick permet le maintien d'Ella à un certain degré de présence et de matérialité. Présence et matérialité y restent donc étroitement associées, alors que la mort d'Ella aurait dû conduire à sa désagrégation physique et par là même à leur dissociation. Les questions que pose Dick ne sont pas dénuées d'intérêt pour notre propos : et si nos moyens d'être présents les uns aux autres se modifiaient? Et si la précognition, la télépathie, etc., étaient des modes de communication à grande échelle? Et si ce monde n'était pas tout à fait aussi tridimensionnel qu'on le prétend? Ses romans regorgent de créatures ambiguës (c'est vrai plus généralement de la science-fiction dans son ensemble) qui ne survivent que parce qu'elles savent se rendre surprenantes, présentes autrement, souvent absentes, ou encore s'abstraire. Elles sont douées d'une grande flexibilité de présence, qui varie entre l'apparition, la rétroprojection et la pseudo-présence (celui avec qui on entre en présence n'est jamais celui qu'on croit). La semi-vie 
admet tout un tas d'hybrides et produit un monde extrêmement chaotique, désordonné, où les connections ne marchent souvent qu'à moitié - comme dans le moratorium, où les câbles sortent des cercueils dans le plus grand désordre. Elle permet à Dick de pousser très loin le fantasme d'entités ayant une flexibilité de présence très étendue, c'est-à-dire au fond une capacité d'occuper des états intermédiaires entre présence et absence, et de naviguer entre des modes, ou degrés, de matérialisation.

L'anthropologie n'a cessé de décrire, comme en témoignent les travaux d'anthropologie religieuse sur les modalités de présence complexes des divinités, des objets semi-présents, à demi absents, jamais totalement matérialisés, jamais complètement absents, des entités caractérisées par un certain flottement, à la fois là et ailleurs. Elle a largement montré la propension des hommes à s'entourer de présences dont le statut est d'être là tout en ne l'étant pas entièrement, des présences qui, si on veut les faire exister, demandent un gros travail, des soins (une idole hindoue n'est vivante que si le prêtre l'a éveillée, sinon elle reste une pierre, seulement potentiellement divine); des entités dont la problématique existentielle se résume au simple fait d'être présentes (ou absentes) ou à faire acte de présence; des entités qui rechignent à se laisser capturer définitivement ou qui, si on les représente, s'échappent ou fuient en partie leur dispositif d'incarnation. Il en est des grands hommes, lorsqu'ils meurent, comme de la plupart des entités trop rapidement qualifiées de «fictives » dont regorge l'anthropologie. On pourrait même faire l'hypothèse que la grandeur d'un être se mesure à la flexibilité de présence ou à l'autonomie d'incarnation qu'il conquiert progressivement après sa mort. Plus un être devient flou dans ses modalités de présence, "flottant", plus il a la capacité de prendre son entourage par surprise et de se rendre imprévisible, plus il a la possibilité d'être à la fois ici et ailleurs, jamais totalement prisonnier d'un seul dispositif, souvent absent (ou abstrait), et plus il a de chances de perdurer.

\section{Des cendres et des sosies}

Que s'est-il passé autour de Gandhi? Après son assassinat en 1948, quelques mois après l'indépendance de l'Inde, par un extrémiste hindou qui voulait signifier ainsi son désaccord face au morcellement du territoire indien résultant de la Partition, Gandhi est incinéré, comme tout hindou, et entre dans le panthéon des icônes de la nation. Sa biographie est bien connue, de même que ses représentations iconiques florissantes, qui ont fait l'objet de plusieurs travaux ${ }^{4}$. Malheureusement, peu d'analyses cherchent à évaluer véritablement la portée des produits dérivés du Mahatma les uns par rapport aux autres, qu'il s'agisse des formes diverses d'imagerie auxquelles il a donné lieu longtemps après sa mort, des sculptures, des automates ou des "images vivantes" qui le représentent encore aujourd'hui sur des chars de parade. On sait, par exemple, que certaines lithographies le dépeignent comme un père de la nation et que d'autres le situent dans des décors divins parmi les avatars du dieu Vishnou (Pinney 2004). On sait aussi qu'il est souvent apparu sur des autels de la fête de Ganesh, le visage crypté dans celui du dieu éléphant (Kaur 2005), qu'il a été le sujet de plusieurs films, de son vivant déjà, et que ses caricatures ont aussi été très nombreuses. Depuis sa mort, sa présence n'a cessé d'osciller, voire d'hésiter, entre différents supports de figuration et le débat autour de ses reliques continue de défrayer la chronique.

\section{- -}

4. Pour une belle biographie de Gandhi, voir Markovitz 2000 - et notamment, pour notre sujet, le troisième chapitre consacré à la vie posthume de Gandhi - et 2004. Sur le contrôle par Gandhi de son image, l'iconographie gandhienne et la place de l'image dans le gandhisme, voir Pinney (2004 et «From portrait to avatar... ») ; et aussi Gell 1998. 
Curieusement, le corps de Gandhi n'a pas subi le même sort que celui de la majorité de ses compatriotes. Ses cendres ont été divisées et envoyées dans les différents États de l'Union indienne pour être dispersées dans toutes les rivières sacrées du pays; mais, pour des raisons assez obscures, une portion a été placée dans une boîte scellée déposée dans une banque de Cuttack, dans l'Orissa. L'arrière-petit-fils de Gandhi, Tushar Arun Gandhi, réclama ces cendres auprès des tribunaux après que des journaux ont révélé l'affaire en 1995. Le reste des cendres du Mahatma fut donc rapatrié au Gujarat et dispersé dans le Gange lors d'une cérémonie officielle le $1^{\text {er }}$ février 1997. C'est Tushar Arun Gandhi qui se chargea d'ouvrir la boîte après avoir enlevé le drap blanc qui la recouvrait et avait jauni avec le temps. En mars 2009, James Otis, collectionneur, cinéaste, activiste de la non-violence et gendre du créateur du Muppet Show, déclara vouloir vendre aux enchères quelques-uns des innombrables objets ayant appartenu à Gandhi qu'il avait en sa possession - dont les lunettes du Mahatma, sa montre à gousset légendaire avec son étui, un bol, une boîte et une paire de sandales. Otis déclara qu'il détenait aussi le rapport médical d'un test sanguin effectué par Gandhi dans un hôpital de Delhi, signé par le docteur B.L. Taneja de l'hôpital Irwin et daté du 21 janvier 1948, c'est-à-dire neuf jours avant son assassinat, ainsi que plusieurs télégrammes griffonnés de la main du Mahatma. Otis confia également qu'un de ses amis, Lester Kurtz, professeur de sociologie et d'anthropologie à l'université George Mason - lui aussi activiste gandhien -, possédait un échantillon des cendres et du sang de Gandhi collectés le jour de son incinération. La collection d'Otis réunissait par ailleurs d'autres témoignages importants de la lutte pour la non-violence : une lettre de Martin Luther King demandant de soutenir le mouvement anti-apartheid en Afrique du Sud, un drapeau des United Farm Workers signé de la main de César Chávez, un peigne et une brosse ayant appartenu à Jane Addams, l'une des premières femmes honorées du prix Nobel de la paix... L'origine de ces divers objets était plutôt floue. Les lunettes de Gandhi semblaient avoir été données en cadeau par le Mahatma lui-même à un colonel anglais de la British Indian Army qui lui aurait témoigné son admiration et à qui le Mahatma aurait dit : "Ces lunettes sont les yeux qui m'ont donné la vision de la libération de l'Inde. » Elles auraient été gardées par la famille du colonel avant d'être cédées à un collectionneur privé. Quant aux sandales, il semblerait qu'en 1931, juste avant la conférence de la Table ronde de Londres à laquelle il participa, Gandhi les ait données à un ami officier de l'armée britannique. Elles se sont retrouvées ensuite dans une collection aux États-Unis. Les autres artefacts, notamment le bol et le plat, auraient appartenu à la petite-nièce du Mahatma, Abha Gandhi, dans les bras de laquelle ce dernier mourut en 1948.

Face aux protestations du gouvernement indien, Otis orchestra la vente de manière plutôt habile, réclamant que l'Inde augmente ses dépenses consacrées à la lutte contre la pauvreté et la violence en échange des «reliques». Celles-ci furent finalement cédées aux enchères à New York pour 1,8 million de dollars à Vijay Mallia, un homme d'affaire indien surnommé "the King of Good Times". Mallia était le propriétaire de la plus célèbre des bières indiennes, Kingfisher, de la ligne aérienne Kingfisher Airlines, de l'écurie de formule 1 Force India et du Grand Jardin, la seule propriété privée de l'île Sainte-Marguerite (en face de Cannes), il était par ailleurs membre du Parlement. En 2003 déjà, Mallia avait acquis dans une vente aux enchères puis rendu à l'Inde l'épée de Tipu Sultan, le gouverneur du royaume de Mysore qui s'est battu au xviII ${ }^{\mathrm{e}}$ siècle contre la Compagnie anglaise des Indes orientales. Quelques jours après les enchères, Otis relança la polémique, réclamant le retour des reliques du Mahatma sous prétexte que 


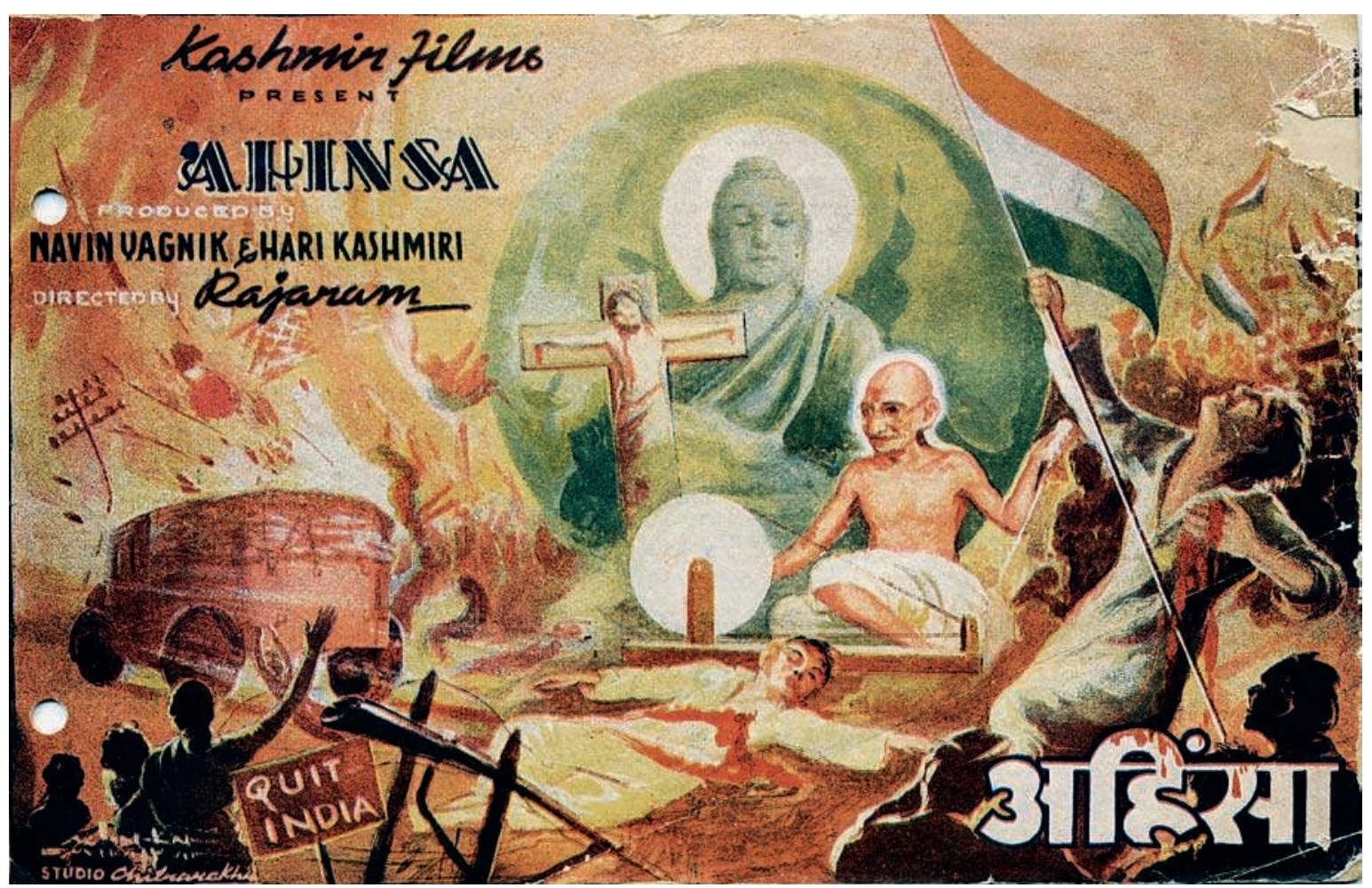

Fig. 3 Ahimsa, Non Violence, affiche d'un film sorti en 1947, quelques mois après l'accès de l'Inde à l'Indépendance, dénonçant les violences de la Partition.

Mallia était un homme politique appartenant au Janata Party, dans l'opposition au parti du Congrès, et que, malgré les déclarations de l'acheteur, il n'était pas du tout sûr que celui-ci les remît sans négociation ou chantage à l'État indien.

La controverse sur les restes matériels et physiques du Mahatma est loin d'être achevée, mais elle possède déjà toutes les composantes d'autres célèbres controverses post mortem, du trésor de Toutankhamon et des disputes autour de sa momie (Grimaud 2003) aux débats les plus récents autour de la tombe de Jésus suscités par la «vraie fausse » découverte de son tombeau. Les cadavres des "grands hommes" ont toujours suscité des jeux étranges. Moins connu, mais sans doute tout aussi signifiant, est le fait que des fidèles de Gandhi ont cherché à le faire exister autrement, bien avant la remise en circulation de ses reliques, en prenant leur distance vis-à-vis de la figuration au sens classique du terme (iconographie, sculpture, film) comme des grandes cérémonies ritualisées célébrant l'Indépendance. Les historiens et les anthropologues qui se sont intéressés à l'iconographie de Gandhi (chromos, photographies, etc.) ont curieusement peu porté leur attention sur les modes de représentation alternatifs que le Mahatma a suscités de son vivant, et encore davantage après sa mort. Alors qu'il était bel et bien en vie, Gandhi eut à faire face à l'irruption d'individus qui adoptèrent sa tenue vestimentaire et propagèrent son message aux quatre coins du pays, prêts à prendre le relais en cas d'épuisement de l'abondante production visuelle dont le Mahatma était le sujet. En sillonnant les campagnes et les villes de l'Inde, Gandhi œuvra, sans le savoir, à sa propre reproduction.

C'est ainsi que dans les années 1980 Kamlesh Jemini, un photographe lié au parti du Congrès, voit surgir dans son studio de Bhopal un homme, Ram Dayal 
Srivastava, fidèle de Gandhi, ancien combattant de l'Indépendance et proche du président de la République Shankar Dayal Sharma, lui aussi originaire de Bhopal. L'homme ressemble étrangement à Gandhi. Il lui dit avoir fait un rêve où Gandhi l'appelait par l'intermédiaire du dieu Hanuman : «Je te donnerai l'apparence de Gandhi, va et accomplis son travail", lui a ordonné le dieu. Pour les gandhiens, l'œuvre du Mahatma n'est pas arrêtée. Elle reste toujours d'actualité et Srivastava est un bon moyen de répandre le gandhisme par un autre moyen, plus physique qu'iconographique. Le photographe prend plusieurs clichés et aide à la promotion de l'homme comme «image vivante» de Gandhi. À partir de ce jour, Ram Dayal Srivastava se rebaptise Ram Gandhi, touche une pension du gouvernement indien en tant qu'« image vivante ", parcourt l'Inde entière et participe à toutes les commémorations officielles liées au Mahatma. On le voit chaque 15 août (jour de la fête nationale) défiler sur un char au milieu des statues et automates de Gandhi, puis le jour du cinquantenaire de l'Indépendance remettre un drapeau de l'Inde à des ministres et à d'importantes personnalités du monde politique. Un producteur de cinéma lui a même proposé de participer à une série télévisée, où il joue son propre rôle. Mais Ram Gandhi se révèle un acteur médiocre - il est d'ailleurs tout sauf un acteur, incapable d'apprendre un texte par cœur, et ne peut être que lui-même devant la caméra, c'est-à-dire un Gandhi légèrement décalé par rapport à l'original. Cela n'a pas empêché les membres du parti du Congrès de Bhopal de vouloir faire de lui un de leurs candidats aux élections, mais Srivastava a repoussé leur offre sous prétexte que le monde politique est corrompu et que Gandhi lui-même avait refusé de son vivant d'occuper tout poste officiel.

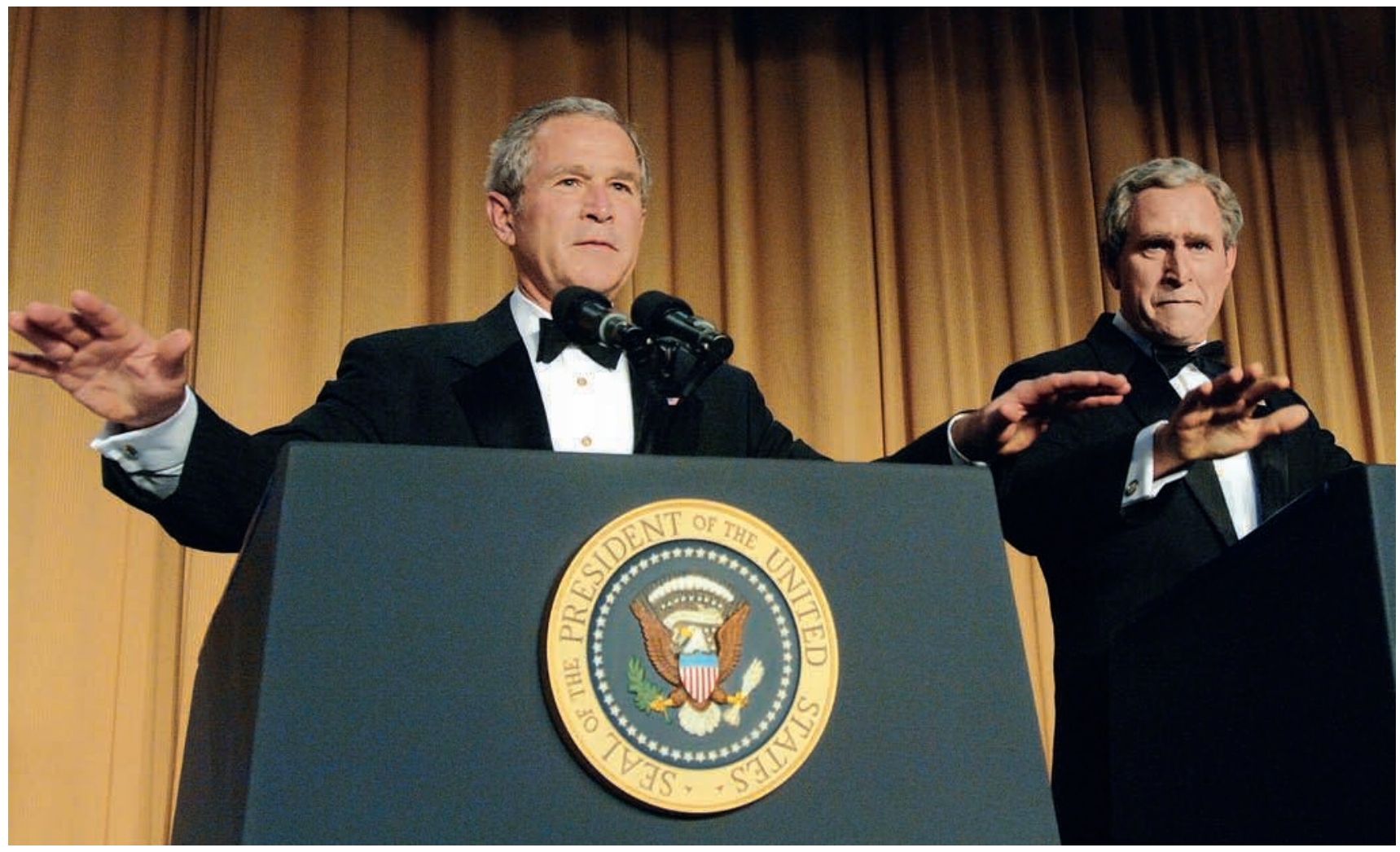

Fig. 4 Georges W. Bush et son sosie Steve Bridges à la Maison Blanche. Dîner annuel des correspondants de presse, Washington, 2006 ๑ Roger L. Wollenberg/Pool/CNP/Corbis. 


\section{Copie humaine d'une photographie}

Je reproduis ici presque en totalité la démonstration du photographe Kamlesh Jemini car elle va nous fournir les clés de la «sosification ». "Ram Gandhi n'est pas un avatar, ni un look-alike, ni un double", dit-il. Mais alors qui est-il? C'est à cette question apparemment simple mais en réalité étrangement compliquée que le photographe apporte une réponse. Quelques années avant la mort de Srivastava en 2004, Kamlesh recompose pour nous la scène où il prit les premières photographies de celui-ci :

"Voici les premières photos que j'ai prises. Je vais tout vous dire », dit Kamlesh en sortant de sa commode quelques photographies, un registre sur lequel il note les visites de ses clients ainsi qu'un livre avec des photographies originales du Mahatma. "C'était le 25 octobre 1988. Si nous n'avions pas les photos de Gandhi, nous n'aurions pas pu photographier M. Ram Dayal Srivastava. Grâce à ma visualisation, l'opération a été possible... J'ai trouvé un indice. " Kamlesh pointe alternativement certains traits du visage du Mahatma sur les photographies et ceux de Ram Gandhi. «Regardez ici, le contour de l'oreille et du crâne qui fait le Gandhi original. Vous les retrouvez chez lui. De longues oreilles et un contour très particulier. Ajoutez-lui des lunettes, et vous y verrez Gandhi en personne! Voilà ce que la photographie et la science des images ont donné au monde. Regardez bien cette oreille qui sort!" Ram Gandhi proteste car Kamlesh manipule sa tête comme une marchandise, mais Kamlesh lui dit de se taire. "Et maintenant regardez son visage en entier! Son visage est un peu plus long que celui du Mahatma, il est ovale alors que celui du Mahatma est rond mais... il ressemble à Gandhi! S'il relève les épaules et que vous le prenez sous cet angle, vous avez exactement Gandhi.» Kamlesh essaie de retrouver la pose du Mahatma qui a servi à élaborer son buste. Il relève l'épaule de Ram Gandhi et me dit de baisser ma caméra pour être à la bonne hauteur. "Stop! C'est ça, c'est le bon angle. " Pause. Kamlesh continue à s'adresser directement à la caméra. "Je n'y suis pour rien, c'est une création de dieu. Après avoir adopté le vêtement du Mahatma, ses ornements, Ram Dayal Srivastava est devenu Gandhi. Mon intention ne fut rien d'autre que de lui montrer ce que j'avais vu en lui. Ce fut ensuite son désir et sa volonté de se conformer à cette vision. Ram Dayal Srivastava est devenu Ram Gandhi. Et quand il prit conscience de sa ressemblance, il eut une révélation. Les gens l'apprécièrent sous cette forme et l'acceptèrent en tant que Ram Gandhi. Ils trouvèrent tout de Gandhi en lui et c'est la raison de sa popularité. Lorsque les cinq ministres reçurent un drapeau de ses mains le jour de la fête de l'Indépendance au Fort rouge de New Delhi, ce fut des mains de Gandhi lui-même, le Bapu. C'est le plus grand honneur que l'on peut recevoir en Inde et je suis fier de l'avoir initié! " Je sens de l'émotion chez Kamlesh. Il est effectivement fier d'avoir réalisé cette prouesse technique et cela se lit dans ses yeux qui brillent. Il se rassied et me demande de me rapprocher de lui avec la caméra avant de reprendre : «Les politiciens se contentent de décorer la statue de Gandhi, mais ils ont oublié ses principes, tandis que M. Ram Dayal Srivastava fait exactement ce que Gandhi recommandait pour le pays! Il peut remplir sans aucun entraînement le rôle dont le parti du Congrès a besoin en ce moment. Ils peuvent très bien l'utiliser, mais ils ne feront pas. Ils ne croient plus à Gandhi, ils préfèrent le célébrer le jour de son anniversaire mais ils n'ont aucun intérêt à promouvoir Ram Dayal Srivastava. » Pause. «Plus que tout, je voudrais insister sur l'idée que Gandhi est le concept de la discipline. Si vous voulez devenir discipliné, vous devez regarder Gandhi, avec 
son bambou qui montre le pouvoir, l'ordre [Kamlesh mime le bâton de Gandhi]; ses lunettes si particulières qui permettent de voir tous les hommes sur un pied d'égalité [il reproduit le tour de ces lunettes avec ses mains]; sa montre qui indique le temps car nous avons perdu le sens du temps; son vêtement en coton qui montre notre pauvreté et ses sandales en caoutchouc qui ne cessent de parcourir les chemins! Ram Dayal Srivastava n'est ni un double de Gandhi ni une photocopie. Il n'est ni un avatar ni le Mahatma lui-même. Je le considère comme la copie humaine d'une photographie.»

Le mot est prononcé. Ram Gandhi est la " copie humaine d'une photographie ", une forme inédite d'être vivant, un nouveau genre de corps plastique. Et Jemini en est l'heureux initiateur. Qu'est-il arrivé exactement dans le studio du photographe? Srivastava a été réduit à une combinaison d'indices visuels, des traits qui ont rendu la duplication de Gandhi possible. Sans ces "points clés », Ram Gandhi n'existerait pas : il serait resté Ram Dayal Srivastava. Ces traits constituent le minimum nécessaire à la reproduction d'un Gandhi. Soit nous acceptons qu'ils suffisent et le corps de Gandhi devient une entité éternellement reproductible, soit nous le refusons et dans ce cas le Mahatma est condamné à survivre sous la forme d'une statue ou d'un cliché photographique, trop sacré pour être retouché, combiné, reproduit et voyager dans d'autres corps humains. C'est après avoir figé Srivastava dans une posture que Jemini fut capable de réanimer Gandhi une nouvelle fois. Dans le studio, le photographe manipule la tête de Srivastava comme s'il auscultait un spécimen rare et se livre face à la caméra à un cours d'anthropologie physique. Il lui demande de s'asseoir, de bouger à droite ou à gauche, de tenir son bâton de bambou bien droit, de sortir sa montre, attirant l'attention sur chaque détail "qui fait un Gandhi ». Il cherche à tout prix à prouver la ressemblance avec les photographies du Gandhi original. L'épreuve est violente, mais terriblement efficace pour faire de Jemini le père de Ram Gandhi. Srivastava proteste à plusieurs reprises, mais le photographe nous rappelle à la cruelle vérité : Ram Gandhi est une photographie faite homme, un cliché animé.

La fin du discours du photographe traduit toute l'intelligence de l'opération, ce que le gandhisme a produit de plus spectaculaire en termes de politique de communication et ce que les studios de photographie ont donné en Inde de plus étonnant pour rattraper le corps humain. Gandhi n'est pas un homme comme les autres, c'est un "concept ", nous dit-il, et un concept peu ordinaire car constitué d'attributs visuels visant à ramener celui qui le contemple à la discipline (le bâton de bambou), à sa pauvre condition (le vêtement), à lui insuffler le sens du temps (la montre), le pouvoir de la marche (les sandales) et à inviter les hommes à voir leurs semblables comme des égaux (les lunettes). C'est sans doute cette mutation de Gandhi en un savant dosage d'attributs ne lui appartenant pas en propre, mais susceptibles d'être la marque de fabrique d'autres hommes à d'autres époques, qui inaugure l'ère de la retouche généralisée de la figure de Gandhi. La "retouche» est sans doute un mot mal choisi car la figure n'a pas vraiment été retouchée au sens d'une correction sur Photoshop, mais elle participe, grâce à sa réduction à quelques traits bien identifiables, à de multiples jeux de recomposition ou combinatoires figuratives. Cette mutation a dû se produire très tôt, du vivant même du Mahatma. Ainsi, dans les années 1930, au Maharahstra, il était courant de trouver en guise de décoration des plateformes rituelles, au moment de la fête de Ganesh, des figures de ce dieu « gandhisées » ou des sculptures de Gandhi « éléphantisées » qui se contentaient de combiner les traits les plus pertinents des deux personnages. Gandhi pouvait donc être présent dans d'autres créatures que lui-même 


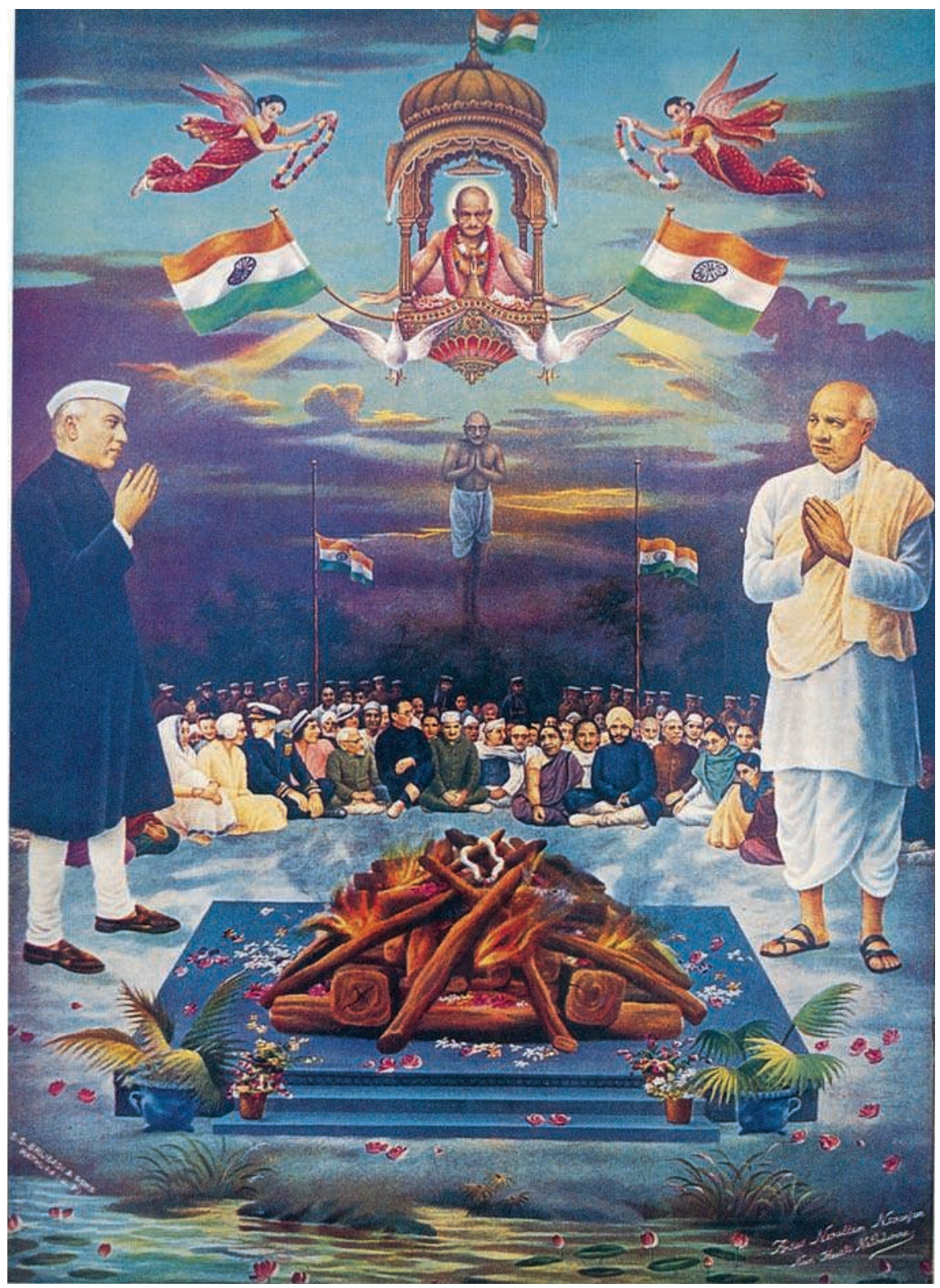

Fig. 5 Gandhiji ki Svargyatra (Montée aux cieux de Gandhi), chromolithographie, 1948, Narottam Narayan Sharma.

uniquement par le jeu d'un rappel de traits indiciels. Il est probable que Jemini s'est au fond contenté de transférer et d'inverser (de la photographie au corps humain) une logique de composition traditionnellement appliquée dans l'autre sens et vers un autre support (du corps humain à la sculpture). Dans la théorie de la représentation implicite du photographe, les concepts sont des éléments corporels (et vice versa), Gandhi est un corps conceptuel et Ram Gandhi le moyen de le rendre vivant, un procédé charnel pour réveiller un public somnolent et le guider vers la discipline. Jemini a réussi le tour de passe-passe qui consiste à faire marcher et parler une photographie en la décomposant en attributs. Et il parvient à nous convaincre que sa créature, ou son «invention ", est une manière de faire de la politique autrement, visant à produire un effet de présence anachronique, 

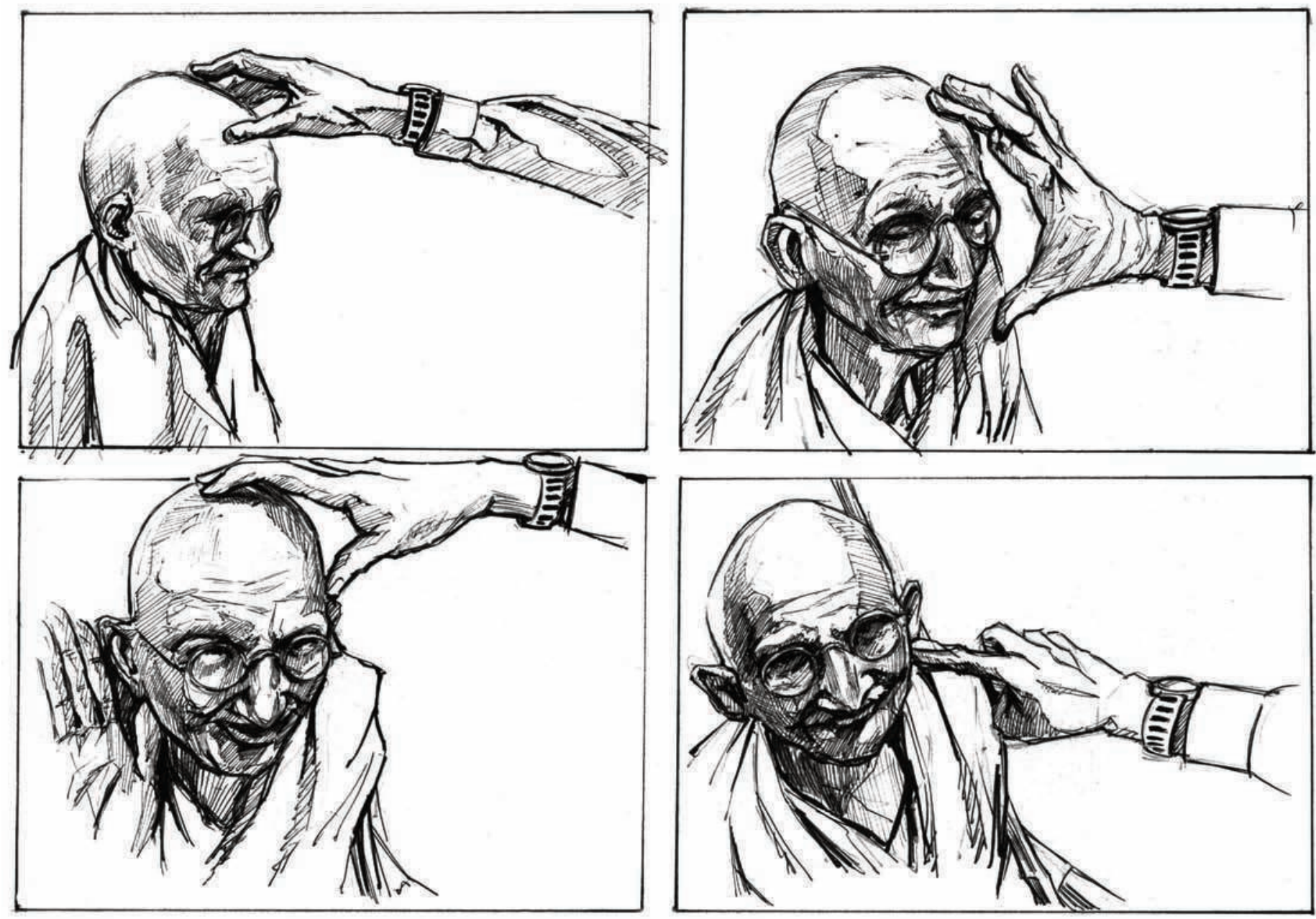

Fig. 6 Démonstration par le photographe Kamlesh Jemini des quelque traits qui suffisent à faire un Gandhi en chair et en os, storyboard du film. Dessin de l'auteur. 


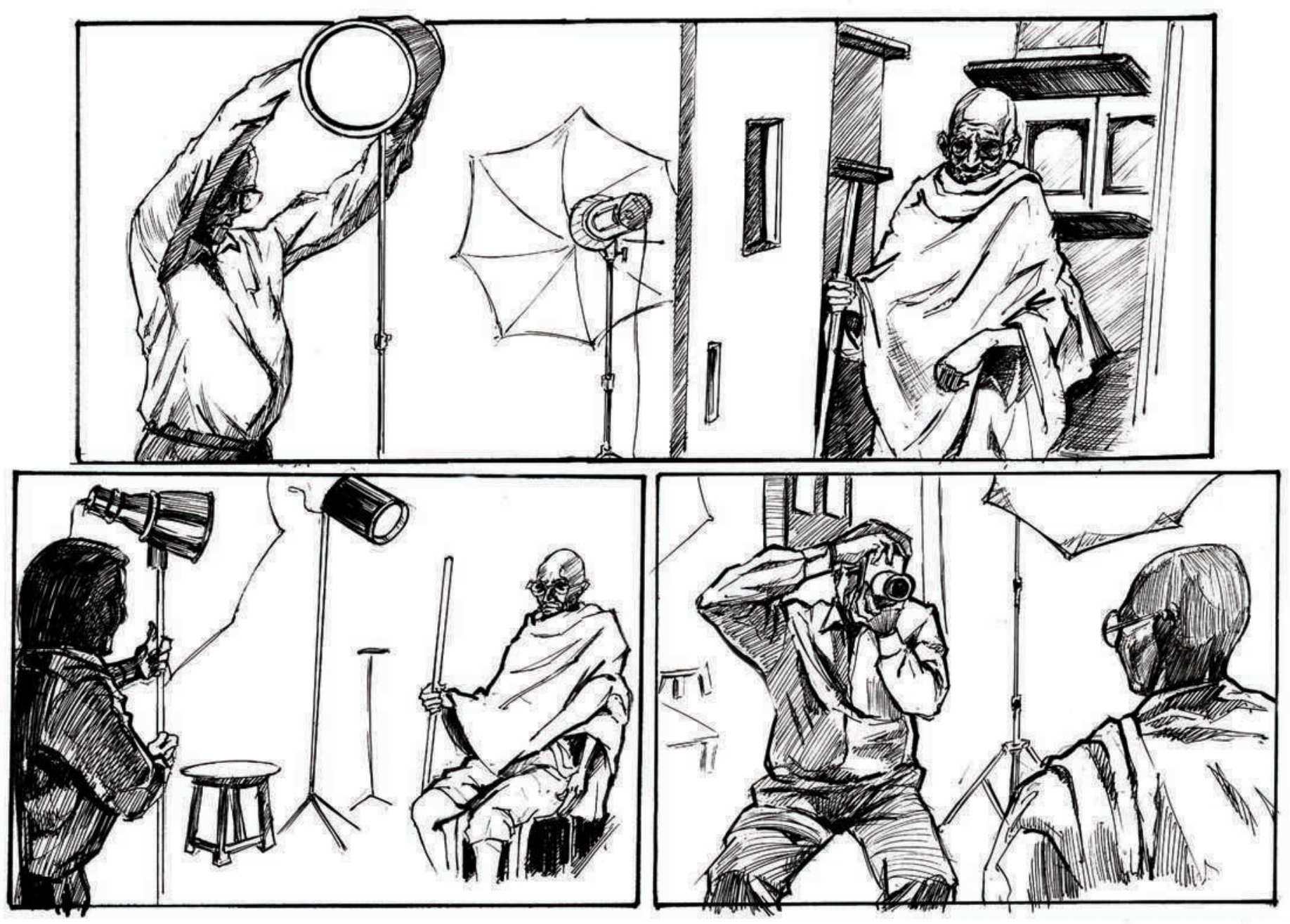

Fig. 7 Le photographe recompose la scène où il révéla au sosie sa ressemblance. Dessin de l'auteur. 
contre l'inauthenticité des hommes politiques qui se réclament de Gandhi sans jamais vivre à sa manière. Ram Gandhi, nous dit-il, est le meilleur exemple, le plus authentique gandhien des hommes politiques existants.

\section{Deux traits + cinq attributs = une nouvelle forme d'anima}

Ainsi, le sosie est arrivé à point nommé pour remédier à une crise du gandhisme, son corps physique venant compenser un déficit de présence, un épuisement de l'iconographie gandhienne. Mais il faut nous attarder un peu plus longuement sur ce que fait le photographe lorsqu'il propose de considérer Srivastava comme la copie humaine d'une photographie fondée sur la reproduction de deux traits (le contour du crâne et celui de l'oreille) et de cinq attributs (lunettes, sandales, tunique blanche, montre, bâton). Pourquoi a-t-il besoin de la photographie pour réaliser cette opération qui consiste à identifier les « indices » de la ressemblance? Et pourquoi tient-il absolument à relier, par un lien quasi consubstantiel, le sosie de Gandhi à la photographie de Gandhi plus qu'à Gandhi lui-même? Si Ram Gandhi était lié à Gandhi par sa seule ressemblance physique, il ne serait qu'un simple sosie, mais grâce à la photographie il est un peu plus que cela : une forme inédite d'anima. Il y a de l'ingéniosité dans l'application que fait le photographe d'une théorie de l'identité finalement assez courante en Inde, selon laquelle une figure est un composé d'attributs, une combinaison subtile de quelques traits (laksana $a^{5}$. Mais Ram Gandhi ne peut être réduit à cela, il nous conduit au-delà de la photographie et des moyens classiques de la représentation. Jemini aurait très bien pu se contenter de relever l'existence du sosie et d'en donner une interprétation en termes de hasard génétique ou encore de coïncidence astrale ou cosmique, sans faire intervenir la photographie ${ }^{6}$, mais il n'aurait pas été fidèle à ce qui s'est vraiment passé dans son studio. Il a révélé Srivastava à lui-même par le biais de l'émulsion photographique. Celui-ci n'est pas un simple sosie, il est la projection d'un Gandhi par photographie interposée, cette dernière ayant servi de négatif au développement du corps du sosie.

Le photographe ne raisonne pas à partir d'un corps atemporel, fait de traits inamovibles, il intègre le fait que, lorsqu'il lui révèle sa ressemblance, Srivastava est une créature évolutive, en développement. Plus ce dernier se voit en photo, plus il se met à ressembler à son modèle. Pour faire émerger ce Gandhi "photo-recomposé", le photographe ne pouvait se contenter de mobiliser la théorie classique des traits décomposant l'identité en attributs. Il fallait réduire l'homme à une image, retourner à la photographie, retrouver la posture, les bons angles, pour prouver sa gandhianité, puis démontrer comment la photographie se dépasse par la projection d'une nouvelle catégorie d'être animé, une sorte de photomorphe, un corps physique imbibé par la photographie et qui sait ce que poser veut dire. D'autant plus que Ram Gandhi surgit après cinquante années d'iconographie gandhienne et de présence photographique hyperréaliste qui ont eu pour résultat de figer le Mahatma. Il est temps de le remettre en mouvement et cela implique un changement de régime plastique. Ainsi, Jemini nous fait parcourir le chemin qui conduit d'une photographie toute plate à un être vivant dans un espace à trois dimensions. Il faut que Ram Gandhi rappelle, lorsqu'il est en mouvement, la fixité de l'iconographie, sans quoi le "photomorphing" de Jemini ne fonctionne pas. Ce n'est qu'une fois aplati, réduit à quelques traits et photographié sous les bons angles que Ram Gandhi acquiert sa dimension et que 
sa sosification est opérante. Si, dans sa démonstration de morphologie appliquée, le photographe doit commencer par bidimensionnaliser le corps du sosie, l'aplatir sous forme de traits, revenir en arrière et remettre en scène la première séance de photographie, c'est parce que ce sosie-là n'est pas un look-alike parfait. Sa ressemblance n'est pas totale, ce qui signifie aussi qu'un sosie possède essentiellement un pouvoir de suggestion, capable de donner l'illusion qu'il est l'original pourvu qu'il ait ces traits minimaux.

Tout, dans la théorie de Jemini, concorde. Il nous dit qu'il n'a rien fait, a simplement révélé l'homme à lui-même, mais il se garde le monopole de cet espace de révélation par la théorie des traits indiciels. Ainsi, Ram Gandhi peut être à la fois, sans contradiction, le fruit d'un peu de coïncidence, une créature artificielle (dont Jemini aurait été l'initiateur), une création divine et un disciple qui serait allé un peu loin (à force d'accompagner Gandhi, de l'écouter et de l'adorer, il aurait fini par lui ressembler); et sa ressemblance est aussi d'une certain manière - c'est ce qu'a bien compris Jemini - dans l'œil de celui qui le côtoie. C'est que, pour qu'un autre Gandhi soit possible, il faut un spectateur, et si possible prêt à faire le travail de reconnaissance. Car certains sosies sont hyper ressemblants, d'autres moins. Certains demandent peu de travail de la part du spectateur pour les identifier tandis que d'autres requièrent un gros effort pour combler les trous et retrouver en eux l'original. Jemini nous a ouvert les yeux sur les traits pertinents, ceux qui font que la reconnaissance est immédiate, mais pas sur l'effet de ressemblance maximal. D'ailleurs, il le dit lui-même : "Sa tête est un peu trop ovale, tandis que celle de Gandhi est ronde, mais... il ressemble quand même à un Gandhi! »

En rapatriant les sosies du côté des créatures mal faites, approximatives, au lieu de les abandonner aux forces du hasard ou au monde des coïncidences heureuses, Jemini a ouvert un champ d'expérimentation immense qui, comme l'affaire des cendres de Gandhi, nous réservera sans doute encore bien des surprises. Avec Gandhi, on l'aura compris, on est à la fois dans la semi-artificialité et la quasi-ressemblance. Si les sosies ne peuvent être appréhendés purement et simplement comme des miracles de la nature et si la sosification d'un individu est effectivement possible, c'est que la ressemblance est avant tout un effet d'optique dont on peut jouer et qui oscille entre la reconnaissance instantanée et l'identification la plus laborieuse. Ce qui est miraculeux, en effet, c'est le processus qui nous conduit à reconnaître une personne dans une autre. Toute une science émerge alors, qui consiste à savoir comment on joue de la quasi-ressemblance, comment celle-ci s'accroît ou s'affaiblit jusqu'à donner une anima de Gandhi en plus ou moins haute résolution, et à partir de quel moment la reconnaissance devient possible ou, à l'inverse, se brouille. La scène précédente rappelle les expériences interactives que font les roboticiens pour tester les réactions des gens devant leurs créatures. Ils savent bien à quel point la viabilité d'un robot dépend des conditions de perception et d'interaction avec ceux qui l'entourent. Les sosies sont, plus que d'autres peut-être, des créatures interactives, qui n'existent que par ce qu'elles suggèrent à ceux qui les perçoivent. Ainsi, en saisissant l'invitation à la reconnaissance que les sosies leur tendent, les individus peuvent prolonger les indices qui leur sont offerts jusqu'à former des figures complètes. D'ordinaire, la ressemblance est incomplète et le sosie une figure en basse résolution; mais les traits minimaux sont un point de départ, à l'entourage du sosie de faire le reste, de l'accompagner et d'orchestrer sa ressemblance, bref de l'aider à exister. 


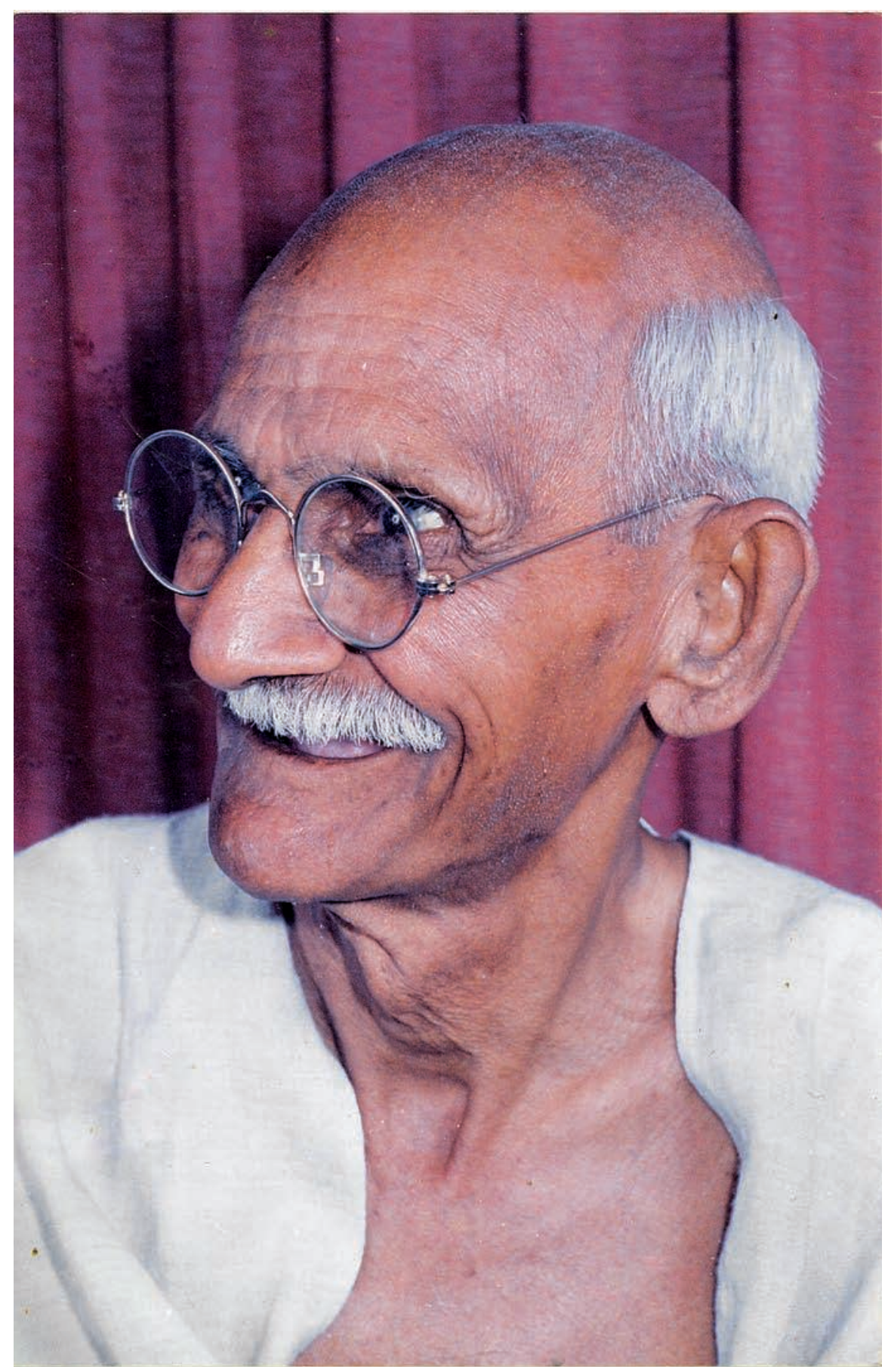

Fig. 8 Photo de Ram Gandhi, issue de ses archives personnelles, cliché K. Jemini. Photo de l'auteur. 
Et si Ram Gandhi était dans le contexte indien un produit d'une extraordinaire banalité? Cela expliquerait qu'on ne se retourne pas dans la rue en le voyant. Un sosie de Gandhi n'attire pas vraiment l'attention ou, en tout cas, pas autant qu'il le devrait si j'en juge par les promenades très ordinaires que nous faisions avec Ram Gandhi. On ne peut pas dire qu'il ait été submergé par les demandes d'autographes ou les commentaires. C'est qu'il n'y a rien d'anormal (ou de paranormal) à ce que Gandhi revienne et se rappelle dans le corps d'un autre homme dès lors que sa réduction indicielle est opérée et acceptée. Mais une fois ces traits en place, il a fallu à Ram Gandhi bien d'autres éléments pour parachever sa stature. Qu'aurait fait en effet le photographe sans la fidélité de Srivastava au projet et au mode de vie de Gandhi? Il n'aurait été rien de plus qu'un manipulateur ou un illusionniste créant une curiosité qui n'aurait eu aucune chance de survie, un véritable "faux», une simple image au fond et non pas un authentique Gandhi en chair et en os. Son studio n'aurait jamais été le laboratoire politique qu'il est devenu. Qu'aurait fait Srivastava sans le miroir du photographe et la possibilité qu'il lui a offerte de se voir comme Gandhi? Son rêve serait resté un rêve parmi d'autres et sa ressemblance n'aurait trouvé aucun écho. Il n'aurait jamais pu être présent sur autant de fronts, multiplier comme il l'a fait marches, meetings et actions de soutien aux causes les plus diverses. Le photographe était là au bon moment pour prolonger et orchestrer comme il le fallait la ressemblance de Srivastava. Il a donné à Ram Gandhi la confiance dont il avait besoin et, sans elle, Srivastava n'aurait pas pu incarner le Mahatma avec la même efficacité.

Et que serait devenu Gandhi sans un Ram Gandhi pour le prolonger dans son existence animée? Il serait resté une statue à décorer, un cliché photographique conservé dans la mémoire des archives, mais sûrement pas une présence aussi vivante. Ainsi, le sosie est suffisamment peu artificiel pour nous faire sortir de la représentation au profit d'une nouvelle politique de la présence. Proche de Gandhi, qu'il a côtoyé, il a plus de valeur en tant qu'extension ou prolongement physique du phénomène gandhien que toutes les peintures, sculptures, projections photographiques ou plastiques que pourraient jamais imaginer les cinéastes, peintres ou sculpteurs. Mais il est aussi suffisamment éloigné de l'original, suffisamment différent pour que des mécanismes classiques de projection se mettent en œuvre. Notre système optique nous pousse à compléter naturellement les images inachevées ou à combler les manques entre deux images qui se suivent. Le sosie de Gandhi «nous conduit vers Gandhi », pour reprendre l'expression du photographe, mais c'est à celui qui le perçoit de compléter le trajet.

Dans un texte intitulé «Un cinéma solide », Paul Mus avait suggéré une thèse intéressante pour expliquer la propension du panthéon hindou à faire des dieux à plusieurs bras et plusieurs têtes. Pour Mus, une statue hindoue composite intègre ainsi à son propre corps, dans une «fusion diachronique ", les différents points de vue qu'un dévot peut avoir alors qu'il fait le tour de la divinité lors de la pérégrination rituelle (pradakshina) : une explication très moderne, de type "chronophotographique» (le corps composé d'un dieu est fait d'une sélection des états de ce dieu dans le temps), d'une énigme que même Hegel, fasciné par l'anthropomorphisme de l'art indien classique, qu'il jugeait «débridé», n'avait pu élucider qu'en faisant basculer l'art indien dans l'irrationalité (1995 [1835]). Peu importe le degré de concordance de l'hypothèse de Mus avec les théories des sculpteurs indiens, certes bien plus anciennes que le cinéma. Elle a l'avantage de suggérer une autre piste pour lire ce qui s'est passé dans le studio de Jemini en termes d'invention plastique. En effet, le photographe réalise là un tour de force, 
en ayant compris et poussé jusqu'au bout l'idée que Gandhi devait être considéré comme une plastique interactive, jusqu'à en faire un principe de recomposition vivante appliqué à un être humain. Si Jemini a pu se permettre d'agir ainsi, sans tabou particulier quant à la recréation du Mahatma, c'est parce qu'il travaillait sur la reconnaissance optique de Gandhi et ses effets, et qu'à aucun moment Ram Gandhi n'a cherché à se faire passer pour ce qu'il n'était pas : la réincarnation de Gandhi lui-même ou son avatar. Il est d'ailleurs remarquable que Ram Gandhi n'ait jamais vraiment suscité de grande polémique, à la différence des cendres de Gandhi ou de ses reliques. Cela ne veut pas dire qu'il n'a pas semé le doute...

\section{Puissance de l'ambiguïté}

Nous avons dit que le sosie de Gandhi ne pouvait être abordé purement et simplement comme une projection, c'est-à-dire au fond comme le produit de l'imagination de son entourage ou le résultat d'un projet politique, celui du gandhisme et de ses initiatives éclatées, ses fondations pour la non-violence, ses entreprises caritatives et ses ventes aux enchères. Ram Gandhi, devenu une "grande âme " par accident (et c'est sans aucun doute ce qui fait sa beauté), lui qui cherchait la libération du cycle des naissances et a réalisé tout le bénéfice karmique qu'il pouvait tirer de sa ressemblance, a trouvé naturellement sa place à la fois comme être indiciel et rappel vivant, visant à stimuler une mémoire du Mahatma toujours présente, mais devenue de plus en plus latente et réticente à s'exprimer avec le temps. Si nous parvenons à lui donner, grâce à la théorie du photographe, une place dans l'histoire des techniques de projection, c'est d'abord parce qu'il a besoin de la photographie comme référence, mais surtout parce qu'il encourage les projections en stimulant les efforts de reconnaissance tout en se plaçant en infraction par rapport à l'histoire des techniques classiques de représentation qui maintiennent les objets dans des cadres délimités.

Tentons de saisir de manière plus large les implications de cette sortie de Gandhi hors du cadre de la représentation au sens classique. Les cadres sont sans doute les dispositifs les plus courants pour faire exister des êtres disparus, et plus largement des entités (devenues) fictives. Leur vision est possible dans des dispositifs bien normés, mais cela ne résout pas le problème de leur présence post mortem, justement parce que ces entités sont peut-être trop optiques dès lors qu'elles n'apparaissent que dans les limites d'un cadre, surtout lorsque celui-ci est un écran. L'innovation technologique peut apporter des outils, multiplier les équipements (des fantasmagories au cinéma) et généralement, pendant une phase provisoire, donner l'impression d'un surplus de résolution, mais souvent, et très vite, celui-ci apparaît insuffisant. Il faut inventer d'autres modes de présence. Ce raisonnement n'est pas le mien, il est celui de tous ceux qui ont cherché à expérimenter d'autres méthodes d'incarnation (après la photographie et le cinéma), et notamment celui des fabricants d'automates indiens, qui ont réagi face à la surenchère des effets spéciaux utilisés par le cinéma mythologique pour rendre les divinités présentes à l'écran. Partant du constat que l'écran de cinéma avait banalisé les apparitions divines en les réduisant à un effet d'optique, les artisans redoublèrent d'ingéniosité pour développer, dans le cadre des plateformes rituelles, une animatronique divine plus économe en mouvements que l'image animée, mais plus physique et aussi plus hypnotisante (Grimaud 2008). Après un siècle de projections cinématographiques offrant des apparitions limitées à 
un cadre bien défini, Ram Gandhi nous rappelle à la puissance du corps animé et nous ramène par la même occasion aux débuts du cinéma. Ainsi, Georges Méliès, entamant sa vie de cinéaste, ne cacha pas sa fascination pour d'autres techniques de mise en présence - et notamment celles du magicien Robert Houdin. Il en racheta d'ailleurs la collection d'automates, comme si un doute s'était installé dès les premières projections sur le déficit d'incarnation d'une image projetée par rapport à celle d'un corps animé.

Tirons les leçons de cette incertitude et tentons de mieux préciser les implications de ce surplus de physicalité du corps animé. Certes, Ram Gandhi est en mouvement alors que les images de Gandhi sont fixes. Il est aussi bien plus flexible et peut se loger dans n'importe quel événement : il suffit de lui téléphoner, de lui dire quand et où il doit apparaître; il est également capable de faire un discours, de saluer les gens et même de plaisanter avec eux. Mais ce n'est pas tout. Il est certain que cette image faite chair avait tout d'un "puzzle visuel», pour reprendre l'expression de James Elkins (1999), et que le meilleur moyen d'entretenir la présence de Gandhi était peut-être au fond de mettre la main sur un être aussi troublant, assez ressemblant mais point trop quand même. Sans pour autant créer la polémique, Ram Gandhi ne pouvait qu'intriguer si l'on en juge par les nombreuses précautions que prend Jemini pour bien nous faire comprendre que Srivastava n'a pas que les traits physiques de Gandhi, mais qu'il en partage aussi les valeurs et le mode de vie. Après tout, le photographe aurait pu se contenter de nous présenter Ram Gandhi sans aucune justification, mais la nécessité même d'une argumentation longue et laborieuse prouve qu'il est conscient que son ami peut provoquer la confusion et être mal interprété.

Le lecteur conviendra aisément que Gandhi existe encore davantage dans un être qui suscite le doute quant à son authenticité et sa parenté avec le Mahatma que dans une créature qui ne soulève aucune interrogation. Les incarnations de Gandhi à l'écran, par exemple, sont fidèles ou infidèles, mais elles ne créent pas le doute quant à l'identité de l'être incarné. Et il en est de même des peintures ou des sculptures, qui au mieux lui sont proches et au pire ne lui ressemblent pas. On pourrait en déduire un principe qui vaut pour de nombreux êtres dont il faut organiser la survivance et qui touche aux modalités de la semi-vie en général. Plus une entité devenue fictive se matérialise, plus s'accroissent à première vue son effet de présence ainsi que son pouvoir d'attraction. Mais arrive un moment où l'effet de présence diminue si on ne le renouvelle pas. L'entité tombe donc dans l'indifférence dès lors qu'elle ne peut muter, que sa présentification ne crée pas le débat et qu'elle perd l'ambiguïté fondamentale qui lui permet de naviguer entre présence et absence. Et, souvent, on n'a pas trop intérêt à la matérialiser davantage sinon elle perd sa qualité fondamentale. Beaucoup des entités dont les anthropologues ont à rendre compte n'existent que si elles sont présentes temporairement ou par inadvertance, ou encore dans des corps qui font que leur existence paraît ambiguë, mais étonnamment flexible. C'est vrai des dieux du panthéon hindou, mais c'est vrai aussi d'autres entités. Et plus il y a d'expérimentations ou d'existences jugées partielles, incomplètes, qu'elles soient plastiques, optiques ou physiques, plus l'entité a de chances de survivre ou de se réincarner autrement, ailleurs, en raison des insuffisances ou des limites de l'enveloppe précédente. On peut donc supposer sans risque que le sosie de Gandhi ne marque pas la fin de l'histoire de Gandhi. Ram Gandhi se devait d'être non seulement un rappel du Mahatma, mais aussi un clin d'œil subtil à l'histoire de ses dispositifs de représentation, dominée par l'iconogra- 
phie, la photographie et la sculpture. L'avenir révélera sans doute qu'il n'en a été qu'une forme partielle dans une courbe de vie post mortem faite d'autres expérimentations plus ou moins performantes; mais le meilleur moyen de garder cette histoire ouverte, du vivant même de Ram Gandhi, a été justement, pour le photographe comme pour son entourage, de reconnaître l'incomplétude ou la partialité de l'anima qu'ils avaient mise au point.

CNRS, LESC e2mgrim@yahoo.fr

motsclés/keywords : ressemblance //similarity•sosie //look-alike •photographies // photographs • représentations // representations $\cdot$ corps // body.

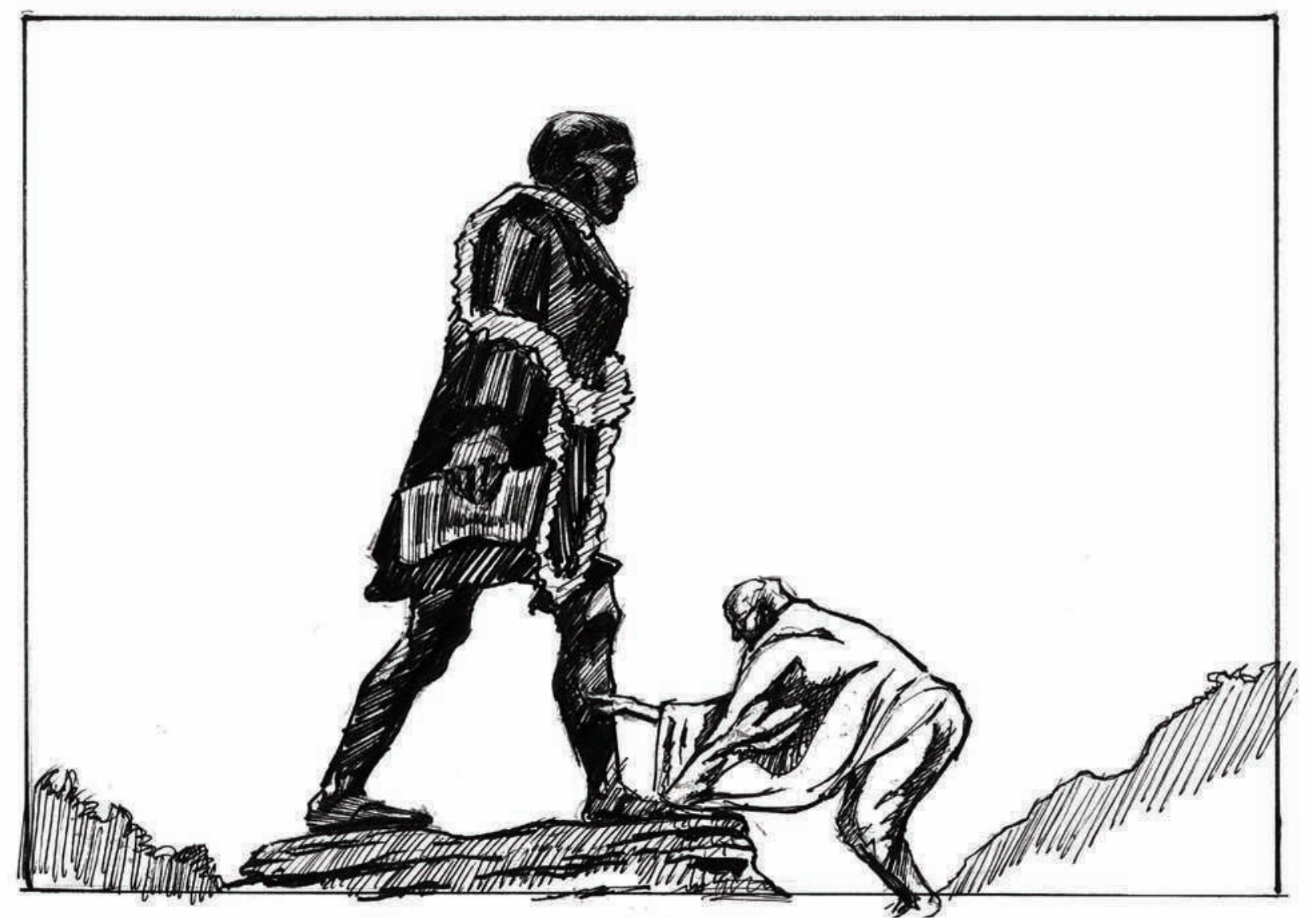

Fig. 9 Ram Gandhi au pied de la statue du Mahatma le jour du cinquantième anniversaire de sa mort. Dessin de l'auteur. 


\section{Bibliographie}

\author{
Dick, Philip K. \\ 1999 [1969] Ubik. Paris, 10/18. \\ DURKHEIM, Émile \\ 1975 [1883] Le Rôle des grands hommes dans \\ l'histoire, rééd. in Émile Durkheim, ćléments \\ d'une théorie sociale. Paris, éditions de Minuit : \\ 409-41?.
}

\section{ELKINS, James}

1999 Why are our pictures puzzles? On the modern origins of pictorial complexity. Londres, Routledge.

\section{GeLL, Simeran}

1998 « L'Inde aux deux visages : Dalip Singh et le Mahatma Gandhi », Terrain 31 : 129-144.

\section{GHoshal SAstri, S.N.}

1983 Elements of Indian aesthetics, vol.3: Indian Gesturology. Bénarès, Chaukhambha Orientalia.

\author{
Grimaud, Emmanuel \\ 2003 « Le trésor de Toutankhamon. Objets, \\ guides et visiteurs au musée d'Art égyptien \\ du Caire », Gradhiva 34 : 15-28. \\ 2007 Le Sosie de Gandhi ou l'incroyable histoire \\ de Ram Dayal Srivastava. Paris, CNRS Éditions. \\ 2008 Dieux et Robots. Les théâtres \\ d'automates divins de Bombay. Apt, \\ L'Archange minotaure.

\section{Hegel, Georg Wilhelm Friedrich} \\ 1995 [1835] Cours d'esthétique, tome I. Paris, \\ Aubier.

KAUR, Ramindar Émile
2005 Performative Politics and the cultures
of Hinduism. Londres, Anthem Press.
MARKOVITZ, Claude
2000 Gandhi. Paris, Presses de Sciences Po.
2004 UnGandhian Gandhi. The Life and Afterlife
of the Mahatma. Londres, Anthem Press.

\section{Mus, Paul}

1964 «Un cinéma solide. L'intégration du temps dans l'art de l'Inde et l'art contemporain », Arts asiatiques (10)1 : 21-34.

\section{Pinney, Christopher}

2004 Photos of the Gods. The printed image and political struggle in India. Londres, Reaktion Books.

«From portrait to avatar: Gandhi in the popular imaginary ». http://www.christopherpinney. com/sitelmages/image/Politics/Gandhi/ gandhirev[1].pdf, article en ligne, dernière consultation décembre 2009.

\section{Sijie, Dai}

2009 L'Acrobatie aérienne de Confucius. Paris, Flammarion.

\section{TARDE, Gabriel}

1902 Psychologie économique. Paris, Félix Alcan (en ligne sur Gallica.fr).

\section{Résumé / Abstract}

Emmanuel Grimaud, Grande âme cherche corps sur mesure. Des animas en général et du sosie de Gandhi en particulier L'histoire d'un sosie de Gandhi chargé de l'incarner après sa mort dans de nombreuses commémorations officielles est le prétexte ici pour aborder un problème anthropologique plus large. La grandeur d'un homme peut-elle se mesurer au nombre de ses figurations, imitations, voire réplications physiques? Et comment évaluer l'intensité de ces techniques d'incarnation les unes par rapport aux autres? Une peinture ne cristallise pas la même chose qu'une sculpture, de même un corps animé par rapport à une image. Le cas exposé invite très concrètement à une telle «matériologie » comparée des modes de présence. Si un corps en chair et en os offre d'autres possibilités qu'une image, il faut adhérer à une théorie plastique bien particulière (révélée au fil de l'enquête) pour que des répliques physiques d'un individu puissent proliférer librement sans être contraintes par le culte de l'original.
Emmanuel Grimaud, Great soul seeks made-to-measure body: of animae in general and, more particularly, of Gandhi's lookalike - After Gandhi's death, a look-alike was employed to stand in for him during a number of official commemorations. This article makes use of these events to discuss a wider anthropological problem. Can the greatness of a man be measured in the number of representations, imitations or even physical replicas of him? How we should gauge the relative intensity of these different techniques of incarnation? A painting does not capture the same thing as a sculpture, nor a moving body the same thing as an image. The case discussed here leads us towards just such a comparative "materiology" of different modes of presence. If a flesh-and-blood body offers different possibilities from an image, then we must adopt a very precise theory of plasticity (progressively revealed over the course of this paper] if a person's physical replicas are to proliferate untrammelled by the cult of the original. 
